\title{
Barriers, Facilitators, Process and Sources of Evidence for Evidence- Based Management among Health Care Managers: A Qualitative Systematic Review
}

\author{
Edris Hasanpoor ${ }^{1,2}$, Sakineh Hajebrahimi ${ }^{3}$, Ali Janati ${ }^{2}$, Zahra Abedini ${ }^{4}$, Elaheh \\ Haghgoshayie $^{5^{*}}$
}

\section{OPEN ACCESS}

Citation: Edris Hasanpoor, Sakineh Hajebrahimi, Ali Janati, Zahra Abedini, Elaheh Haghgoshayie. Barriers, Facilitators, Process and Sources of Evidence for Evidence-Based Management among Health Care Managers: A Qualitative Systematic Review. Ethiop J Health Sci.2018;28 (5): 665 .

doi:http://dx.doi.org/10.4314/ ejhs.v28i5.18 Received: February 14, 2018

Accepted: January February 20, 2018

Published: September 1, 2018

Copyright: (C) 2018 Edris H., et al. This is an open access article distributed under the terms of the Creative Commons Attribution License, which permits unrestricted use, distribution, and reproduction in any medium, provided the original author and source are credited.

Funding: Nil

Competing Interests: The authors declare that this manuscript was approved by all authors in its form and that no competing interest exists.

Affiliation and Correspondence:

Department of Healthcare

Management, Maragheh University of Medical Sciences, Maragheh, Iran

${ }^{2}$ Iranian Center of Excellence in Health Management, School of Management and Medical Informatics, Tabriz University of Medical Sciences, Tabriz, Iran

${ }^{3}$ Research Center for Evidence Based

Medicine, Tabriz University of Medical Sciences, Tabriz, Iran

${ }^{4}$ Department of Health Management and Economics, School of Public Health, Tehran University of Medical Sciences, Tehran, Iran

${ }^{5}$ Department of Healthcare Management, School of Nursing and Midwifery, Maragheh University of Medical Sciences, Maragheh, Iran

*Email: ezd_ehm2010@yahoo.com

INTRODUCTION

\section{ABSTRACT}

BACKGROUND: Evidence-based management (EBMgt) improves managerial decisions as a bridge from theory to practice. For reason that it has a critical impact on organization performance. The purpose of this study was to identify factors affecting EBMgt among managers.

METHODS: The following electronic databases were used: PubMed, Web of Science, Cochrane, ProQuest, Embase and Scopus. In addition, we searched Google Scholar, Emerald, Academy of Management (AOM), and the website for the Center for Evidence-Based Management (CEBMa) for articles related to EBMgt. We used data sources published up to September 2017, without language restriction. We appraised the methodological quality of studies using the checklists of SRQR and MMAT. The synthesis involved interpretative analysis based on the principles of meta-synthesis.

RESULTS: Of 26,011 identified studies, 26 met the full inclusion criteria. Of the 26 studies assessed, the frequency of qualitative studies and mixed-methods were 20 and 6 , respectively, and the quality of 3 studies was weak. A total of 23 studies from 7 countries were included: Canada $(n=8), U S A(n=6)$, Australia $(n=4)$, UK $(n=3)$, Iran (n=1, Brazil $(n=1)$; none were from Africa. Meta-synthesis findings of 23 studies identified four main factors: facilitators (5 main themes), barriers (5 main themes), sources of evidence (4 main themes), and the process of decision making in EBMgt (1 main theme).

CONCLUSIONS: EBMgt is crucial to improve the quality of management decisions, and hence, to improve service delivery, effectiveness and efficiency. Furthermore, to increase the benefit and utilization of EBMgt, training organizations and research institutes must more actively involve managers in setting research plans.

KEYWORDS: Evidence-based management, evidence-based medicine, healthcare organizations 
There has been an intense effort toward developing modern models for organization and administration, especially in the last 20 years. One of these models is evidence-based management (EBMgt) for managing different organizations. Originally, the concept of EBMgt was derived from evidence-based medicine (EBM). Analogous to EBM, evidence in EBMgt serves as a tool to solve problems about the likely result of a decision (1-6).

EBMgt is an evolution in the practice of management and organizations. There have been debates regarding the adoption of EBMgt with criticism since 1998, when EBMgt was in its early stages. While the theory of EBMgt is fairly youthful, it has become increasingly popular over the past few years (1-3).

EBMgt is imports making decisions through the accurate, clear and judicious utilization of the best available evidence from multiple sources to increase the likelihood of a suitable outcome $(1,2$, 7). Therefore, managers have a responsibility to make effective and efficient decisions which help the mission and vision of their organization, similar to physicians, who utilize the best available scientific evidence in clinical decisions about patients (8-10).

Researchers and managers have emphasized the demand for enhanced consideration and mobilization of evidence-based decision making (EBDM) to support management actions in organizations. In point of fact, we live in a period of "evidence-based" everything, and that everything-medicine, management, disaster management, nursing, organizations and hospitalshave become information-based. What matters is managers makes the managerial decisions according to the best available evidence. Hence, using evidence to aid management development and practice in organizations has earned high excellence $(1-4,11-14)$.

EBMgt means making decisions about managing organizations through the conscientious, explicit and judicious use of four multiplex sources. The four sources of evidence for management decision-making include the best available scientific evidence, organizational evidence, experiential evidence and stakeholders' and patient's expectations (1-3). The use of evidence sources in decision-making processes among healthcare administrators has the potential for a more productive, cost-effective, high quality and efficient healthcare organizations $(1,2,15$, 16).

EBMgt has been slowly adopted by healthcare managers in the USA, the UK and Canada (16-18). However, a remarkable gap exists between this ideal scenario and the status quo (19, 20). The major factors were identified from the different studies. EBMgt or EBDM have been affected by several factors, including the organizational factors, facilitators, barriers, strategies plus individual and social factors (11, 21, 22). Guo (2015) identified the strongest predictors of EBMgt among 154 healthcare managers in the USA. The results showed that it was important to create a culture and receive organizational reinforcement in the practice of EBMgt (16). Alavi et al. conducted a study about managers' awareness of EBDM. The results showed that managers' awareness of EBDM in the hospitals was $3.08 \pm 1.13$ (score range $=0-6$ ) (15).

There are many theories regarding EBMgt. These theories have been inspired by researchers and experts of management and organization. Axelsson, Konver, Walshe and Rundall, Pfeffer and Sutton, Rousseau, Briner, Barends, Edris and Wright have all reviewed the concept, application and components of EBMgt in their studies in recent years $(10,23-29)$.

The purpose of this review was to identify factors and components of the EBMgt based on a systematic review. Therefore, we wish to provide a practical framework for EBMgt, based on recent evidence. This framework is appropriate for managing health sectors, hospitals, industries and every organization. Therefore, we wish to provide a practical framework for EBMgt, based on recent evidence. Identifying factors affecting EBMgt and designing the final framework of EBMgt is a new perspective for managing organizations and can be a new skill, practices and behavior.

\section{METHODS}

DOI: http://dx.doi.org/10.4314/ejhs.v28i5.18 


\section{Search strategy and selection criteria}

The search was formulated using the following broad parameters:

Types of participants: In this review, studies were included that healthcare managers (men and women) worked at healthcare institutions or organizations who had used experiences of evidence-based management. The entire healthcare managers at all levels of management (operational, middle and senior) in healthcare organizations were included.

Phenomena of interest: The studies were included that focus on the experiences and perceptions of healthcare managers who had experienced evidence-based management approach. Studies focusing on healthcare managers' experiences and perceptions of nonevidence-based management were excluded.

Type of context: The context of the review was healthcare institutions or organizations (any type of institution/organization) including all levels of management from any setting globally.

Types of studies: The inclusion criteria were as follows:

- Primary studies (English) were included

- Qualitative studies, including (but not limited to), phenomenological, grounded theory, ethnographic, case studies and thematic analysis studies of healthcare managers' experiences and perceptions of evidence-based management were included.

Studies that were not related to manager, administrator, director and other managerial posts were excluded. Also, quantitative studies were excluded. The systematic review and meta- synthesis were performed and reported according to the standards set out in Preferred Reporting Items for Systematic Reviews and Meta-Analyses (PRISMA) (30). The following electronic databases were searched: PubMed, Web of Science, Cochrane, ProQuest, Embase and Scopus. In addition, we searched Google Scholar, Emerald, Academy of Management (AOM) and the website for the Center for Evidence-Based Management (CEBMa) for articles related to EBMgt. On the other hand, references of relevant articles were checked that were not found in searching databases.

We used data sources up to September 2017, without language restriction. In addition, we updated searches through Google Scholar and alert system of databases up to December 2017. The search strategies for the databases combined subject terms: Evidence based management [Title/Abstract], Evidence informed management [Title/Abstract], Evidence based decision making [Title/Abstract], Evidence informed decision making [Title/Abstract], Evidence based policy making [Title/Abstract], Evidence informed policy making [Title/Abstract], Evidence based administration [Title/Abstract], Evidence informed administration [Title/Abstract], Evidence based health [Title/Abstract], Evidence informed health [Title/Abstract], Evidence based organization [Title/Abstract], Evidence informed organization [Title/Abstract], Evidence based hospital [Title/Abstract], Evidence informed hospital [Title/Abstract], Organization [Title/Abstract], Administration[Title/Abstract], Hospitals[MeSH Terms] and Evidence based practice [MeSH Terms]. 


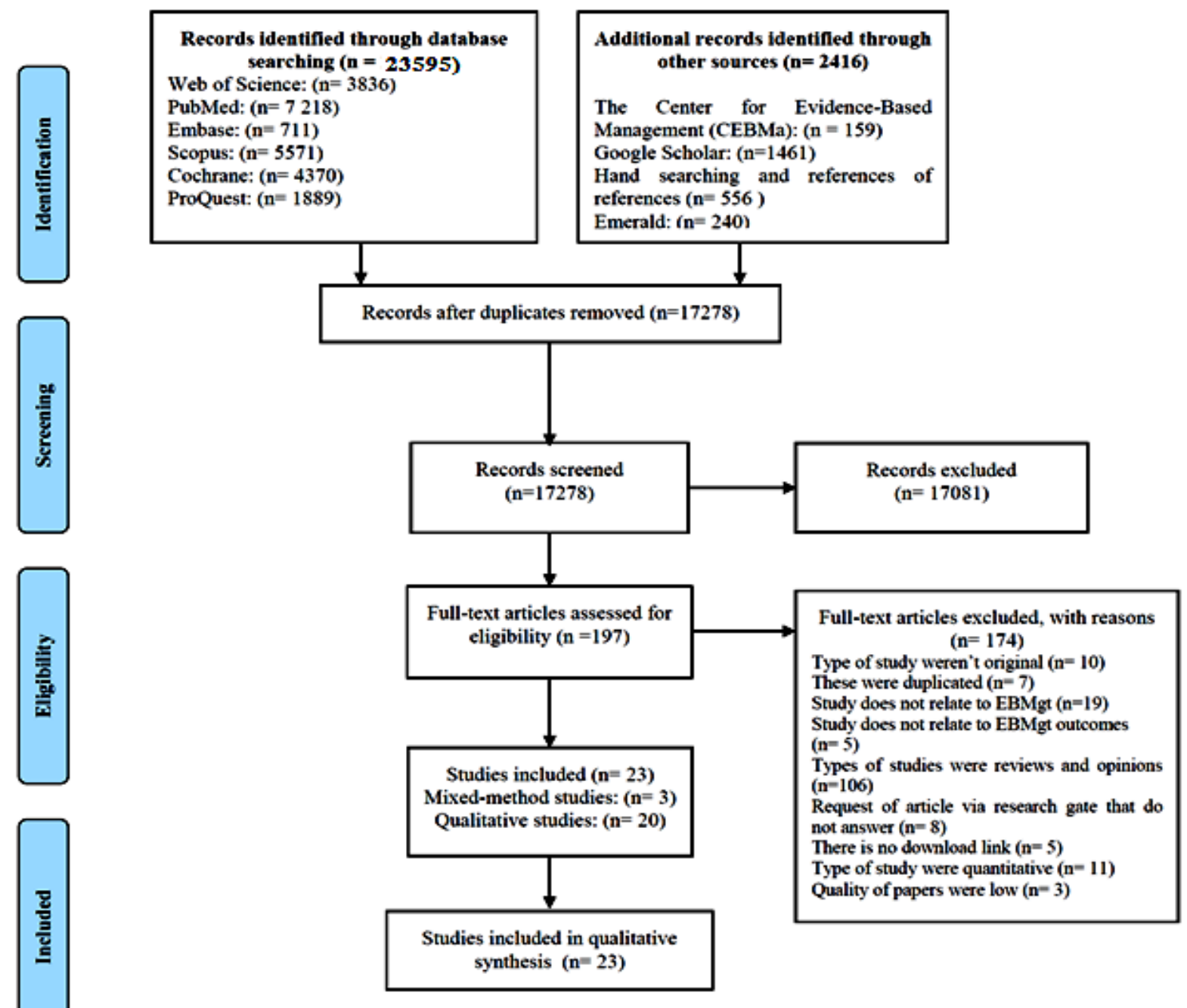

Figure 1: Flow diagram of selected studies

Quality assessment and data extraction: We appraised the quality of qualitative and mixedmethod studies using the SRQR (Standards for Reporting Qualitative Research) (31) and MMAT (Standards for Reporting Qualitative Research) (32), respectively. All of the phases, including searching, screening, quality assessment, and data extraction, were reviewed by two independent researchers (EH and $\mathrm{EHG})$, and the discrepancies were resolved by a third researcher (MAZ). We extracted data per country, aim, design, method of data collection, analysis, participants, context, main outcome and rating quality scores of quality assessments. The quality of studies was categorized into the following levels: strong, moderate and weak.

Data synthesis: The synthesis involved interpretative analysis following the principles of meta-synthesis. Articles were read, re-read, and details of the studies were recorded. Data extraction forms were used to record details of results coded as first and second order bodies (3335). First order constructs are study participants' explanations of their experience (direct quotes from participants); second order constructs are 
studied authors' interpretations of the participants' accounts. Data extraction forms were used by thematic coding according to key articles and continued through all 23 articles. Also, the synthesis was a cyclical process; when a new theme was identified, we returned to the other articles to survey the theme event.

\section{RESULTS}

Among the 26,011 records identified from database searches, 17,278 remained after we removed duplicates, and 197 articles remained for full-text assessment. Overall, 26 studies were selected $(11,15,18,19,21,22,29,36-65)$. However, 174 studies were excluded. Of the 26 studies assessed, the frequency of qualitative studies and mixed-methods were 20 and 6 , respectively; the quality of 3 studies was weak. Finally, 23 studies were included in the Metasynthesis.

A total of 23 studies from 7 countries were included: Canada $(\mathrm{n}=8)$, USA $(\mathrm{n}=6)$, Australia $(\mathrm{n}=4)$, UK $(\mathrm{n}=3)$, Iran $(\mathrm{n}=1)$ and Brazil $(\mathrm{n}=1)$; none were from Africa. The period of included studies ranged from 2003 to 2016. Most studies were conducted in the context of healthcare sector.
Of 174 articles excluded, 106 were review and opinion articles.

The quality levels of studies were designated as strong, moderate and weak. The results of quality assessment showed that 20(53.84\%) articles were of high quality, and $9(34.62 \%)$ were of moderate quality. The quality level of 3 studies was low (11.54\%).

Included studies were 23, of which 20 were qualitative studies and 6 were mixed-methods (Table 1). The main themes and outcomes presented are evidence research in EBMgt, facilitating factors, EBMgt training, barriers and facilitators to implementing supports for EBMgt, role of evidence in the decision-making of EBMgt, components of EBMgt, EBMgt decision process, and implications for designing EBMgt and factors of evidence-based decision making (EBDM). Meta-synthesis was conducted as per four main outcomes and was categorized into the following factors: facilitators ( 5 main themes), barriers ( 5 main themes), and sources of evidence (4 main themes) and the process of EBDM (one main theme). The results of synthesis are shown in Table 2. 
Et670p J Health Sci.

Vol. 28, No. 5

September 2018

Table 1: Included Studies Characteristics $(\mathrm{n}=23)$

\begin{tabular}{|c|c|c|c|c|c|c|c|c|}
\hline Author/year & Country & Aim & Design & $\begin{array}{l}\text { Method of } \\
\text { data collection }\end{array}$ & Analysis & Participants (n) & Context (n) & $\begin{array}{c}\text { Quality of } \\
\text { paper }\end{array}$ \\
\hline $\begin{array}{l}\text { Alexander et } \\
\text { al., } 2007\end{array}$ & USA & $\begin{array}{l}\text { To identify the relevance of } \\
\text { research tohealth care managers }\end{array}$ & Qualitative study & $\begin{array}{l}\text { Semi-structured } \\
\text { interviews }\end{array}$ & $\begin{array}{l}\text { Content } \\
\text { analysis }\end{array}$ & CEOs $(n=8)$ & $\begin{array}{l}\text { Public, profit and non- } \\
\text { profit Hospitals }(n=8)\end{array}$ & Moderate \\
\hline $\begin{array}{l}\text { Amodeo et al., } \\
2013\end{array}$ & USA & $\begin{array}{l}\text { To identify Facilitating Factors } \\
\text { in implementing evidence based } \\
\text { practice }\end{array}$ & Qualitative study & $\begin{array}{l}\text { Semi-structured } \\
\text { telephone }\end{array}$ & $\begin{array}{l}\text { Content } \\
\text { analysis }\end{array}$ & Staff and administrators $(\mathrm{n}=$ & $\begin{array}{l}\text { Substance Abuse and } \\
\text { Mental Health Services } \\
\text { Administration }\end{array}$ & Moderate \\
\hline $\begin{array}{l}\text { Bullock et al., } \\
2012\end{array}$ & UK & $\begin{array}{l}\text { To evaluate Collaboration } \\
\text { between health services } \\
\text { managers and researchers }\end{array}$ & $\begin{array}{l}\text { Qualitative study } \\
\text { (case study) }\end{array}$ & $\begin{array}{l}\text { Semi-structured } \\
\text { face-to-face } \\
\text { interviews }\end{array}$ & $\begin{array}{l}\text { Thematic } \\
\text { analysis }\end{array}$ & $\begin{array}{l}\text { Health services managers } \\
\qquad(\mathrm{n}=10)\end{array}$ & NHS & Strong \\
\hline $\begin{array}{l}\text { Champagne et } \\
\text { al., } 2014\end{array}$ & Canada & $\begin{array}{l}\text { to assess } \\
\text { whether and how the training of } \\
\text { mid- and senior-level healthcare } \\
\text { managers could lead to } \\
\text { organizational change }\end{array}$ & $\begin{array}{l}\text { Qualitative study } \\
\text { (case studies) }\end{array}$ & Interviews & $\begin{array}{l}\text { Thematic } \\
\text { analysis }\end{array}$ & $\begin{array}{l}\text { Healthcare leaders } \\
\qquad(\mathrm{n}=84)\end{array}$ & $\begin{array}{l}\text { health systems of } \\
\text { Alberta, Saskatchewan, } \\
\text { Quebec and Nova } \\
\text { Scotia }\end{array}$ & Strong \\
\hline $\begin{array}{l}\text { M. E. Ellen et } \\
\text { al., } 2013\end{array}$ & Canada & $\begin{array}{l}\text { What supports do health system } \\
\text { organizations have in place to } \\
\text { facilitate evidence informed } \\
\text { decision making }\end{array}$ & Qualitative study & $\begin{array}{l}\text { semi-structured } \\
\text { telephone } \\
\text { interviews }\end{array}$ & $\begin{array}{l}\text { Thematic } \\
\text { analysis }\end{array}$ & $\begin{array}{l}\text { Senior managers, team } \\
\text { member, library manager } \\
\text { and knowledge broker } \\
\qquad(\mathrm{n}=57)\end{array}$ & $\begin{array}{c}\text { Healthcare } \\
\text { organizations }(n=25)\end{array}$ & Strong \\
\hline $\begin{array}{l}\text { Moriah E. } \\
\text { Ellen et al., } \\
2014\end{array}$ & Canada & $\begin{array}{l}\text { To identify barriers and } \\
\text { facilitators to implementing } \\
\text { supports for EBDM }\end{array}$ & Qualitative study & $\begin{array}{l}\text { semi-structured } \\
\text { telephone } \\
\text { interviews }\end{array}$ & $\begin{array}{l}\text { Thematic } \\
\text { analysis }\end{array}$ & $\begin{array}{l}\text { Senior management and } \\
\text { knowledge broker }(\mathrm{n}=57)\end{array}$ & $\begin{array}{c}\text { Health care } \\
\text { organizations }(n=42)\end{array}$ & Strong \\
\hline $\begin{array}{l}\text { Francis- } \\
\text { Smythe et al., } \\
2013\end{array}$ & UK & $\begin{array}{l}\text { The role of evidence in general } \\
\text { managers' decision-making }\end{array}$ & Qualitative study & $\begin{array}{l}\text { Semi-structured } \\
\text { interviews }\end{array}$ & $\begin{array}{l}\text { Thematic } \\
\text { analysis }\end{array}$ & Senior managers $(n=29)$ & $\begin{array}{l}\text { Public and private } \\
\text { organizations }(n=5)\end{array}$ & Moderate \\
\hline $\begin{array}{l}\text { Reza } \\
\text { Majdzadeh et } \\
\text { al., } 2012\end{array}$ & Iran & $\begin{array}{l}\text { To identify barriers to EBDM in } \\
\text { Iran's health system }\end{array}$ & Qualitative study & $\begin{array}{c}\text { in-depth } \\
\text { interviews } \\
(\mathrm{n}=13), \text { FGDs } \\
(\mathrm{n}=6)\end{array}$ & $\begin{array}{l}\text { Thematic } \\
\text { analysis }\end{array}$ & $\begin{array}{l}\text { Policy-makers, managers of } \\
\text { the Ministry of Health and } \\
\text { Medical Education } \\
\text { (MOHME) }(\mathrm{n}=13)\end{array}$ & $\begin{array}{l}\text { Ministry of Health and } \\
\text { Medical Education }\end{array}$ & Strong \\
\hline McBride, 2015 & USA & $\begin{array}{l}\text { To provide a framework for } \\
\text { EBDM based on the } \\
\text { experiences of the research } \\
\text { participants }\end{array}$ & $\begin{array}{l}\text { Qualitative study } \\
\text { (phenomenology) }\end{array}$ & $\begin{array}{l}\text { Interviews } \\
\quad(\mathrm{n}=15)\end{array}$ & $\begin{array}{l}\text { Content } \\
\text { analysis }\end{array}$ & Office managers $(n=15)$ & $\begin{array}{c}\text { Financial services and } \\
\text { Health care }\end{array}$ & Strong \\
\hline
\end{tabular}

DOI: http://dx.doi.org/10.4314/ejhs.v28i5.18 
Table 1. Continued...

\begin{tabular}{|c|c|c|c|c|c|c|c|c|}
\hline $\begin{array}{l}\text { Peirson et al., } \\
2012\end{array}$ & Canada & $\begin{array}{c}\text { To explore factors and } \\
\text { dynamics for building } \\
\text { evidence informed decision } \\
\text { making capacity }\end{array}$ & $\begin{array}{l}\text { Qualitative study } \\
\text { (case study) }\end{array}$ & $\begin{array}{c}\text { Semi-structured } \\
\text { interviews }(n=6) \text { and } \\
\text { FGDs }(n=21)\end{array}$ & $\begin{array}{l}\text { Thematic } \\
\text { analysis }\end{array}$ & $\begin{array}{l}\text { Library personnel, directors, } \\
\text { managers, supervisors and } \\
\text { specialist }(\mathrm{n}=70)\end{array}$ & Public health unit & Strong \\
\hline Plath, 2013 & Australia & $\begin{array}{l}\text { To illustrate the } \\
\text { implementation of evidence- } \\
\text { based practice as an } \\
\text { organizational change process }\end{array}$ & $\begin{array}{l}\text { Qualitative study } \\
\text { (case study) }\end{array}$ & $\begin{array}{c}\text { Semi-structured } \\
\text { interviews }(\mathrm{n}=24) \text { and } \\
\text { Focus Group } \\
\text { Discussions }(\mathrm{n}=5)\end{array}$ & $\begin{array}{l}\text { Content } \\
\text { analysis }\end{array}$ & $\begin{array}{l}\text { Senior executives, Senior } \\
\text { staff, State and area } \\
\text { managers, Team leaders and } \\
\text { Clinical specialists }(n=24)\end{array}$ & $\begin{array}{c}\text { National } \\
\text { organizations } \\
(n=2)\end{array}$ & Moderate \\
\hline Plath, 2014 & Australia & $\begin{array}{c}\text { To illustrate the model of } \\
\text { evidence based practice in } \\
\text { organizations }\end{array}$ & $\begin{array}{l}\text { Qualitative study } \\
\text { (case study) }\end{array}$ & $\begin{array}{l}\text { Semi-structured } \\
\text { interviews }(n=24) \text { and } \\
\text { FGDs }(n=5)\end{array}$ & $\begin{array}{l}\text { Content } \\
\text { analysis }\end{array}$ & $\begin{array}{l}\text { Senior executives, Senior } \\
\text { staff, State and area } \\
\text { managers, Team leaders and } \\
\text { Clinical specialists }(n=24)\end{array}$ & $\begin{array}{c}\text { National } \\
\text { organizations }\end{array}$ & Moderate \\
\hline $\begin{array}{c}\text { Richer et al., } \\
2013\end{array}$ & Canada & $\begin{array}{l}\text { To examine the notions of } \\
\text { evidence in decision-making } \\
\text { processes in health care }\end{array}$ & Qualitative study & $\begin{array}{c}\text { Individual semi- } \\
\text { structured interviews }\end{array}$ & $\begin{array}{l}\text { Content } \\
\text { analysis }\end{array}$ & $\begin{array}{l}\text { Decision makers and } \\
\text { managers }(\mathrm{n}=11)\end{array}$ & $\begin{array}{c}\text { McGill } \\
\text { University Health } \\
\text { Centre }\end{array}$ & Strong \\
\hline Scheller, 2014 & USA & $\begin{array}{l}\text { To analyze the } \\
\text { implementation of an } \\
\text { organizational change } \\
\text { initiative (EBL) }\end{array}$ & $\begin{array}{c}\text { Qualitative study } \\
\text { (comparative case } \\
\text { study) }\end{array}$ & $\begin{array}{l}\text { in-depth qualitative } \\
\text { interviews }\end{array}$ & $\begin{array}{l}\text { Content } \\
\text { analysis }\end{array}$ & $\begin{array}{l}\text { Leaders and managers of } \\
\text { hospital and short health } \\
\text { system }(n=30)\end{array}$ & $\begin{array}{l}\text { Long hospitals } \\
\text { and short health } \\
\text { system }\end{array}$ & Strong \\
\hline $\begin{array}{l}\text { Sosnowy et } \\
\text { al., } 2013\end{array}$ & USA & $\begin{array}{l}\text { To determine use of decision } \\
\text { making processes by } \\
\text { leaders and identify } \\
\text { facilitators and barriers to the } \\
\text { use of evidence based } \\
\text { decision making }\end{array}$ & Qualitative study & $\begin{array}{l}\text { Individual interviews } \\
(\mathrm{n}=20), \text { FGDs }(\mathrm{n}=2) \\
\text { and small-group } \\
\text { interviews }(\mathrm{n}=5)\end{array}$ & $\begin{array}{l}\text { Thematic } \\
\text { analysis }\end{array}$ & $\begin{array}{l}\text { Decision makers (Upper- } \\
\text { level staff) of Local health } \\
\text { departments }\end{array}$ & $\begin{array}{l}\text { Local health } \\
\text { departments } \\
\qquad(n=31)\end{array}$ & Moderate \\
\hline $\begin{array}{l}\text { Spiri and } \\
\text { MacPhee, } \\
2013\end{array}$ & Brazil & $\begin{array}{c}\text { To understand the meaning of } \\
\text { EBMgt to Brazilian senior } \\
\text { nurse leaders }\end{array}$ & $\begin{array}{l}\text { Qualitative study } \\
\text { (phenomenology) }\end{array}$ & $\begin{array}{l}\text { Individual semi- } \\
\text { structured interviews }\end{array}$ & $\begin{array}{l}\text { Content } \\
\text { analysis }\end{array}$ & Senior nurse leaders $(\mathrm{n}=10)$ & $\begin{array}{l}\text { Public hospitals } \\
\qquad(\mathrm{n}=10)\end{array}$ & Strong \\
\hline $\begin{array}{l}\text { Wright, } \\
\text { Zammuto, et } \\
\text { al., } 2016\end{array}$ & Australia & $\begin{array}{c}\text { To fill the gap in knowledge } \\
\text { about the process and } \\
\text { particularities of EBMgt }\end{array}$ & $\begin{array}{l}\text { Qualitative study } \\
\text { (case study) }\end{array}$ & $\begin{array}{c}\text { Semi-structured } \\
\text { interviews }(n=29)\end{array}$ & $\begin{array}{l}\text { Inductive } \\
\text { procedures }\end{array}$ & $\begin{array}{c}\text { Emergency } \\
\text { physicians } \\
\text { and registrars }(\mathrm{n}=24), \\
\text { CEOs }(\mathrm{n}=4) \text { and nurse } \\
(\mathrm{n}=1)\end{array}$ & Hospitals & Strong \\
\hline
\end{tabular}


Et672p J Health Sci.

Vol. 28, No. 5

September 2018

Table 1. Continued...

\begin{tabular}{|c|c|c|c|c|c|c|c|c|}
\hline $\begin{array}{l}\text { Ferlie et al., } \\
2012\end{array}$ & UK & $\begin{array}{l}\text { To consider implications for } \\
\text { designing a more modest } \\
\text { project for EBMgt in health } \\
\text { care organizations }\end{array}$ & $\begin{array}{l}\text { Qualitative study } \\
\text { (case study } \\
\text { methods) }\end{array}$ & Individual interviews & Paired analysis & $\begin{array}{c}\text { General and clinical } \\
\text { managers Phase 1: } \\
(\mathrm{n}=45) \text { Phase } 2: \\
(\mathrm{n}=45)\end{array}$ & $\begin{array}{c}\text { Health care } \\
\text { organizations }(n=6)\end{array}$ & Moderate \\
\hline Kohn, 2013 & Canada & $\begin{array}{c}\text { To explores how evidence is } \\
\text { conceptualized by public } \\
\text { hospital } \\
\text { Executives }\end{array}$ & $\begin{array}{l}\text { Qualitative study } \\
\text { ( A grounded } \\
\text { theory approach) }\end{array}$ & Individual interviews & Content analysis & $\begin{array}{c}\text { CEOs, } \\
\text { healthcare leaders, } \\
\text { decision makers } \\
(\mathrm{n}=18)\end{array}$ & $\begin{array}{c}\text { Public hospitals }(n=4), \\
\text { academic health sciences } \\
\text { centers }(n=2) \text { and teaching } \\
\text { hospitals }(n=2)\end{array}$ & Strong \\
\hline $\begin{array}{c}\text { Jack et al. } \\
2011\end{array}$ & Canada & $\begin{array}{c}\text { To describe the } \\
\text { types and sources of } \\
\text { information used to inform } \\
\text { practice related } \\
\text { decisions }\end{array}$ & $\begin{array}{l}\text { Qualitative study } \\
\text { (descriptive) }\end{array}$ & $\begin{array}{l}\text { In-depth semi- } \\
\text { structured interview }\end{array}$ & Content analysis & $\begin{array}{c}\text { Executive } \\
\text { directors }(n=8), \\
\text { Program managers } \\
(\mathrm{n}=12), \text { and service } \\
\text { providers }(\mathrm{n}=6)\end{array}$ & Canadian agencies $(n=24)$ & Strong \\
\hline $\begin{array}{l}\text { Armstrong } \\
\text { et al., } 2014\end{array}$ & Australia & $\begin{array}{l}\text { Describes how evidence is } \\
\text { used to inform local } \\
\text { government (LG) public } \\
\text { health decisions }\end{array}$ & $\begin{array}{l}\text { Mixed -method } \\
\text { (cross-sectional } \\
\text { survey and } \\
\text { interview) }\end{array}$ & $\begin{array}{l}\text { Semi-structured } \\
\text { telephone } \\
(\mathrm{n}=13)\end{array}$ & $\begin{array}{c}\text { Descriptive } \\
\text { statistics and } \\
\text { content analysis }\end{array}$ & CEOs $(n=135)$ & Local government $(n=45)$ & Moderate \\
\hline $\begin{array}{l}\text { Martelli, } \\
2012\end{array}$ & USA & $\begin{array}{l}\text { To support the construct of } \\
\text { knowledge variety of } \\
\text { evidence-based management } \\
\text { for organizations. }\end{array}$ & $\begin{array}{l}\text { Mixed-method } \\
\text { (cross-sectional } \\
\text { survey and } \\
\text { interview) }\end{array}$ & $\begin{array}{l}\text { Questionnaire and } \\
\text { semi-structured } \\
\text { interviews }\end{array}$ & $\begin{array}{l}\text { Descriptive } \\
\text { statistics } \\
\text { and content } \\
\text { analysis }\end{array}$ & $\begin{array}{l}\text { CEOs, CAOs, CFOs, } \\
\text { CIO/CTO, CMO, } \\
\text { CNO, CHO }(\mathrm{n}=103)\end{array}$ & Hospitals $(\mathrm{n}=42)$ & Moderate \\
\hline $\begin{array}{l}\text { Yost et al., } \\
2014\end{array}$ & Canada & $\begin{array}{l}\text { To evaluate the impact of an } \\
\text { intensive education } \\
\text { workshop on Evidence } \\
\text { informed decision making } \\
\text { knowledge }\end{array}$ & $\begin{array}{l}\text { An explanatory } \\
\text { mixed- methods }\end{array}$ & $\begin{array}{l}\text { Individual semi- } \\
\text { structured interview } \\
(\mathrm{n}=8), \text { Questionnaire }\end{array}$ & $\begin{array}{c}\text { Descriptive } \\
\text { statistics and } \\
\text { content analysis }\end{array}$ & $\begin{array}{c}\text { CEOs, } \\
\text { Associate medical } \\
\text { officer of health, } \\
\text { program manager and } \\
\text { care provider }(\mathrm{n}=42)\end{array}$ & $\begin{array}{l}\text { University and health care } \\
\text { organizations }\end{array}$ & Strong \\
\hline
\end{tabular}

Abbreviations:

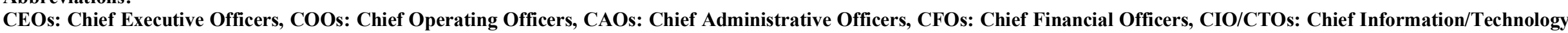
Officers, CMOs: Chief Medical Officers, CNOs: Chief Nursing Officers, HNs: Head Nurses, HDDs: Health Departments Directors 
Table 2: Meta- Synthesis of Studies $(n=23)$

\begin{tabular}{|c|c|c|}
\hline Factors affecting EBMgt & Main themes & Sub-themes \\
\hline \multirow{5}{*}{$\begin{array}{l}\text { Facilitators of EBMgt (n= } \\
\text { 13) } \\
\text { Amodeo et al., 2013; } \\
\text { Armstrong et al., 2014; } \\
\text { Champagne et al., 2014; } \\
\text { M. E. Ellen et al., 2013; } \\
\text { Moriah E. Ellen et al., } \\
\text { 2014; Jack et al., 2011; } \\
\text { Peirson et al., 2012; } \\
\text { Plath, 2013; Richer et } \\
\text { al., 2013; Schuller et al., } \\
\text { 2015; Sosnowy et al., } \\
\text { 2013; Spiri and } \\
\text { MacPhee, 2013; Wright, } \\
\text { Zammuto, et al., 2016. }\end{array}$} & $\begin{array}{l}\text { Organizational } \\
\text { factors }\end{array}$ & $\begin{array}{l}\text { Compensation and reward system, organizational and administrative support, clear vision, workforce } \\
\text { development, organizational structure, a receptive organizational culture, create a knowledge translation } \\
\text { culture, developing and implementing an infrastructure, organizations programs of EBMgt, promotion of } \\
\text { staff development opportunities, time frame for making decisions, recognize in recruitment and retention } \\
\text { strategies, emphasize the value of research use, build awareness of clear points of contact, place value on } \\
\text { accreditation components, knowledge intelligence service, publish and disseminate local research results, } \\
\text { institute communications and marketing efforts related to research evidence, quality and safety standards, } \\
\text { organizational processes and local connections. ( } 21 \text { factors) }\end{array}$ \\
\hline & $\begin{array}{l}\text { characteristics and } \\
\text { individual factors }\end{array}$ & $\begin{array}{l}\text { attitude, strong leadership, knowledge management, effective communication, genuine interest, access to } \\
\text { research evidence, focus on change management, training and continuing education of EBMgt, ensure } \\
\text { decision-making processes, participatory decision-making, responsibilities, previous exposure to research, } \\
\text { self-belief, rationality, determination and expertise in tailoring communication, recognized need (for change), } \\
\text { insider trust and art of judgment. ( } 22 \text { factors) }\end{array}$ \\
\hline & $\begin{array}{l}\text { Factors related to } \\
\text { research } \\
\text { productions }\end{array}$ & $\begin{array}{l}\text { Participate in the production of primary research, reviews and research-derived products, funding for priority } \\
\text { projects, priority-setting processes, ensure research commissioning capacity and use dedicated staff to pull } \\
\text { research, summarize or conduct primary research and presentation of evidence and interactive workshops. ( } 7 \\
\text { factors) }\end{array}$ \\
\hline & $\begin{array}{l}\text { External or } \\
\text { environmental } \\
\text { factors }\end{array}$ & $\begin{array}{l}\text { Grant and regulatory requirements, buy-in from local government, availability of evidence-based } \\
\text { programming suitable to local conditions, regulations and policies, community, councilors, council size and } \\
\text { structure and statutory focus. ( } 8 \text { factors) }\end{array}$ \\
\hline & $\begin{array}{l}\text { Social / } \\
\text { interpersonal } \\
\text { factors }\end{array}$ & $\begin{array}{l}\text { Integrated team, group norms/socialization, stimulus, interest from the management, collaboration between } \\
\text { managers and researchers, personal commitment to EBMgt, participatory decision-making, magnitude of the } \\
\text { decisions, building trust between researchers and managers, use of opinion leaders to promote practice, hold } \\
\text { regular meetings, establish formal and informal ties to researchers and brokers, evaluation efforts to link } \\
\text { research to action and training of skills development of EBMgt. (14 factors) }\end{array}$ \\
\hline Barrier to EBMgt $(n=6)$ & Decision-makers & Lack of criteria for selecting decision-makers, lack of reward and incentive mechanism, Insufficient \\
\hline $\begin{array}{l}\text { Armstrong et al., 2014; } \\
\text { Moriah E. Ellen et al., }\end{array}$ & characteristics & $\begin{array}{l}\text { knowledge and negative attitude toward EBMgt, lack of trust in domestic evidence, lack of awareness of } \\
\text { researchers' ability, excuse of lack of time to make true evidence based decisions. ( } 7 \text { factors) }\end{array}$ \\
\hline $\begin{array}{l}\text { 2014; Ferlie et al., 2012; } \\
\text { Reza Majdzadeh et al., } \\
\text { 2012; Plath, 2013; Spiri } \\
\text { and MacPhee, 2013. }\end{array}$ & $\begin{array}{c}\text { Decision-making } \\
\text { environment }\end{array}$ & $\begin{array}{l}\text { EBMgt is not an organizational value, limited outlook in decisions, influence of non-technical issues, } \\
\text { capacity of policy implementation environment, lack of EBDM's influence on budget allocation, resistance } \\
\text { to innovation, lack of co-ordination between decision-making organization sectors and concern of public } \\
\text { perception supersedes evidence. ( } 8 \text { factors) }\end{array}$ \\
\hline
\end{tabular}

DOI: http://dx.doi.org/10.4314/ejhs.v28i5.18 
Table 2. continued...

Training and

research system

Organizational

barriers

Team barriers

Sources of evidence

$$
(n=9)
$$

Ellen et al., 2013;

Francis-Smythe et al.,

2013; Jack et al., 2011;

Kohn, 2013; Oliver,

2013; Richer et al., 2013;

Sosnowy et al., 2013;

Spiri and MacPhee,

2013; Wright,

Zammuto, et al., 2016.

EBMgt Process $(n=3)$

McBride, 2015; Plath,

2014; Wright,

Zammuto, et al., 2016. evidence to managers

EBMgt decision making
Not having systematic health research prioritization, resource constraints, lack of communication between knowledge producers and decision-makers, time to look for evidence, uncertainty of the evidence base confidence in using research, lack of development of skills in finding, accessing and using, lack of research and evaluation skills, lack of accessibility of management research, lack of transference of knowledge and lack of EBMgt education. (11 factors)

bureaucracy and power dynamics within traditional organizational hierarchies, social and historical trends that impede innovation uptake and utilization, Organizational culture opposed to EBMgt, limited resources, lack of time, workloads, lace of competing priorities, lack of leadership commitment, lace of regulations and policies and lack of understanding by leadership. (10 factors)

Resistance to change, resistance to the source of evidence, presence of inexperienced leaders and negative attitude toward change. (4 factors)

Organizational Organizational data, internal data, facts, extensive personal networks inside, social or political mandates,

External evidence agency mandate and resources, agency service providers and agency clients. ( 8 factors)

Scientific evidence from the professional literature, reports from other organizations to benchmark or compare with their own, Research evidence, tools, frameworks, and models to use with their data, extensive personal networks beyond, webinars, seminars and conference. (10 factors)

Evidence related Trial and error, personal values, leaders' knowledge of the organization, its employees, and patient

Types of evidence population; formal education, previous experience, instinct and common sense. ( 7 factors)

Research evidence, best practice guidelines, perceived best practices, local program evaluations, client needs assessments, expert opinion, personal professional experiences and an individual's personal experiences of addiction and recovery. ( 8 factors)
1. Define and redefine practice questions, 2. Gather evidence, 3. Critically appraise evidence, 4. Engaging stakeholders and generating evidence based alternatives, 5. Committing to an evidence-based solution and implementation, 6. Evaluate EBMgt process and client outcomes. (6 factors) 


\section{DISCUSSION}

The evidence shows that organizational managers have a positive attitude towards EBMgt $(36,39)$. On the other hand, managers' use of evidence sources showed that 94 percent of managers utilized from personal experience (36). The major constraint of EBMgt was a lack of time $(11,39,40$, 43). Alavi et al. conducted a study in Iran and showed that training influence on the level of manager's awareness. $(\mathrm{P}<0.01)(15)$. Predictors of administrative evidence-based practices in the local health departments in the US were categorized into the following areas: factors of workforce development, factors of leadership, organizational climate and culture, relationships and partnerships, and financial processes that 50 percent of directors agreed with it (37).

Levels of access to evidence to a range of resources, levels of confidence in searching, assessing the quality and synthesizing the sources of evidence, and organizational culture are essential to support the EBMgt.(63) EBMgt is essential in progressing the quality of manager's decisions, and hence, improved service delivery, effectiveness, and efficiency in health care organizations $(2,3,43)$.

Everything in organizations has become evidencebased $(2,3)$. This is a claim of EBMgt. Thus, the practical framework of EBMgt should be designed based on the best available evidence. In this study, the factors affecting EBMgt were identified among organizational managers that factors were categorized into the following domains: facilitators, barriers, sources of evidence, and process of EBMgt decision making. The practical framework of EBMgt was designed based on the exploratory factors of different studies. The framework will guide managers of various organizations that they make the best decisions.

The evidence-based organization is a system that its management uses the practical framework of EBMgt. As shown in Figure 2, initially the barriers and facilitators must be identified per sources of evidence (best evidence) for implementing EBMgt. Therefore, facilitators must be supported, and barriers must be converted to facilitators or should be eliminated. When the infrastructure of the organization and management is provided, organizational and managerial decisions will be made using cycle of EBDM during six steps (6A). In the cycle of EBDM, the pyramid of evidence must be considered to make best decisions based on available best evidence. In the beginning, practical issues or problems must be translated into an answerable question and then systematically searched and retrieved. The third step is critically judging trustworthiness while the fourth step is weighing and pulling the evidence. Finally, evidence must be applied to the decisionmaking process. Then, outcome of the decision evaluated. The pyramid of evidence show levels of evidence that help to make the best decisions.

It must be noted that the practical framework of evidence-based management should be based on the best resources from identifying barriers and facilitators. To date, meta-analyses and meta-syntheses (meta-meta), based on RCTs studies, were included the highest level. A question mark (?) will be stronger evidence than meta-meta in future studies.

It must be noted that EBMgt is not related to a specific period, but always looking for the best evidence. EBMgt should be taught by professional coaches and then used by managers and leaders. EBMgt is the art of using the best for achieving the best. Several factors have played different roles in affecting the practice of EBMgt among healthcare managers. The interaction between these factors is complex. Thus, the framework developed in this study may guide the development of strategies to encourage and improve the utilization of evidence in management decision-making process. Furthermore, to increase the benefit and utilization of EBMgt, training organizations, universities, healthcare centers and research institutes must more actively involve hospital managers in setting research plans. Also, it is essential that appropriate presentation of research evidence should be fully considered to facilitate the interpretation of research evidence created to improved management practice in the health care organizations. 


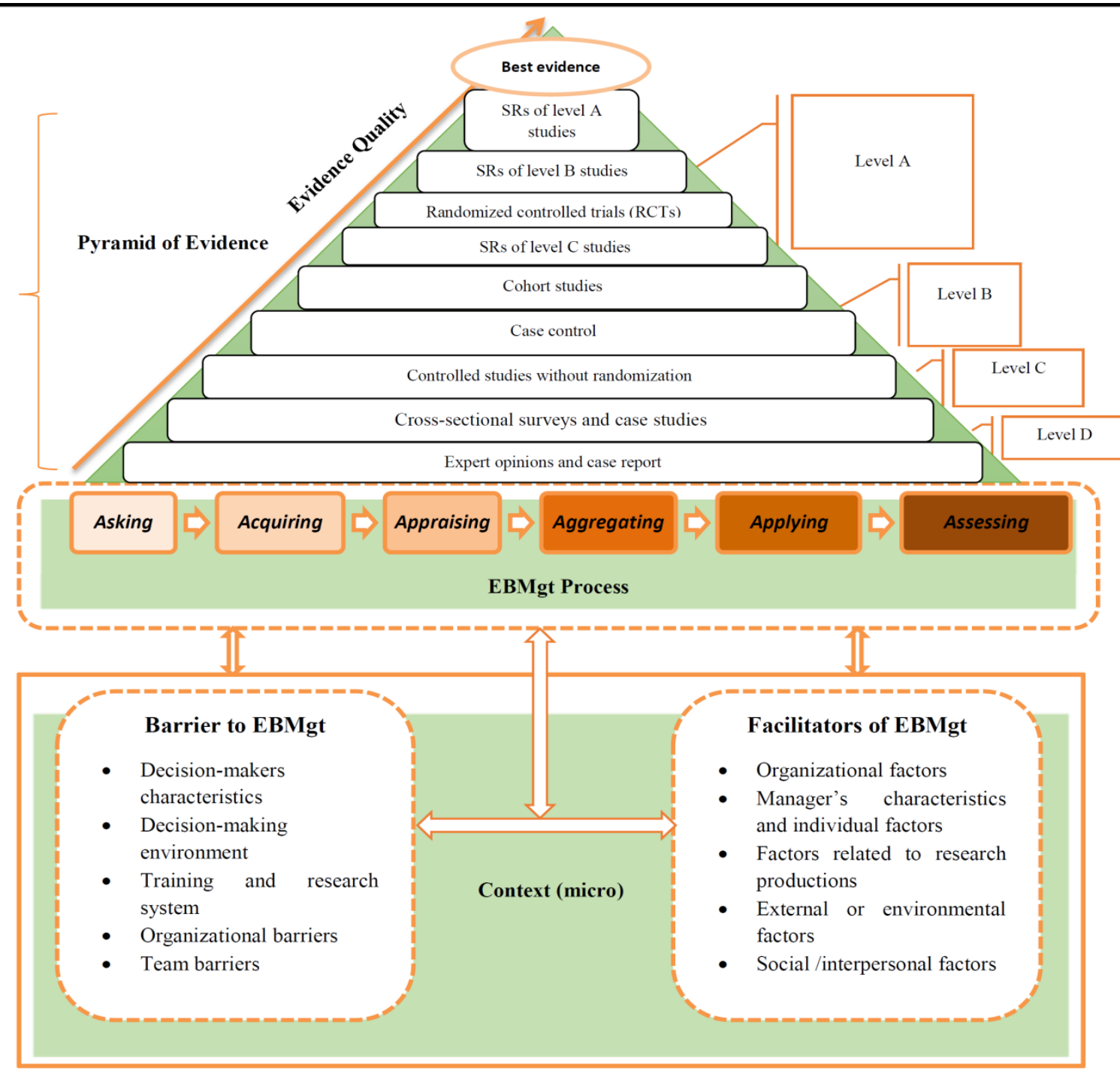

Context (macro): Government, healthcare organizations, training organizations and research institutes

Figure 2: The practical framework of EBMgt

\section{ACKNOWLEDGEMENTS}

This study was based on an evaluation approved by the Deputy of Research Affairs at Tabriz University of Medical Sciences. We are grateful to Research Center for Evidence Based Medicine at Tabriz University of Medical Sciences.

\section{REFERENCES}

1. Janati A, Hasanpoor E, Hajebrahimi S, Sadeghi-Bazargani H. Health care managers' perspectives on the sources of evidence in evidence-based hospital management: A qualitative study in Iran. Ethiopian journal of health sciences. 2017;27(6):659-68. 
2. Janati A, Hasanpoor E, Hajebrahimi S, Sadeghi-Bazargani H. Evidence-based management-healthcare manager viewpoints. International Journal of Health Care Quality Assurance. 2018;31(5):436.

3. Janati A, Hasanpoor E, Hajebrahimi S, Sadeghi-Bazargani H, Khezri A. An Evidence-Based Framework for EvidenceBased Management in Healthcare Organizations: A Delphi Study. Ethiopian journal of health sciences. 2018;28(3):30514.

4. Janati A, Sadeghi-Bazargani H, Hasanpoor E, Sokhanvar M, HaghGoshyie E, Salehi A. Emergency response of Iranian hospitals against disasters: a practical framework for improvement. Disaster medicine and public health preparedness. 2018;12(2):166-71.

5. Janati A, Sarabchian MA, Mohaghegh B, Aghmohamadzadeh N, Seyedin H, Gholizadeh M, et al. Health economic evaluation of home and hospital-based care in T2D patients on insulin therapy. Ethiopian journal of health sciences. 2017;27(6):651-8.

6. Tabrizi JS, Goshayie EH, Doshmangir L, Yousefi M. The Barriers to Implementation of New Public Management Strategies in Iran's Primary Health Care: A Qualitative Study. Journal of the liaquat university of medical and health sciences. 2018;17(1):817.

7. Kovner AR, Rundall TG. Evidence-Based Management Reconsidered. Frontiers of Health Services Management. 2006;22(3):3-22.

8. Wright AL, Middleton S, Greenfield G, Williams J, Brazil V. Strategies for Teaching Evidence-Based Management: What Management Educators Can Learn From Medicine. Journal of Management Education. 2016;40(2):194-219.

9. Sackett DL, Rosenberg WM, Gray JM, Haynes RB, Richardson WS. Evidence based medicine: what it is and what it isn't. BMJ (Clinical research ed). 1996;312(7023):71-2.

10. Walshe K, Rundall TG. Evidence-based management: From theory to practice in health care. The Milbank Quarterly. 2001;79(3):429-57.

11. Liang Z, Howard P. Evidence-informed managerial decision-making: What evidence counts?:(Part two). Asia Pacific Journal of Health Management. 2011;6(2):12.

12. Liang Z, Howard PF, Leggat SG, Murphy GB. A framework to improve evidenceinformed decision-making in health service management. Australian Health Review. 2012;36(3):284-9.

13. Lohr KN, Carey TS. Assessing "best evidence": issues in grading the quality of studies for systematic reviews. Joint Commision Journal on Quality Improvement [Internet]. 1999; (9):[470-9]. Available from: http://onlinelibrary.wiley.com/o/cochrane/c lcmr/articles/1344/frame.html.

14. McAlearney AS, Garman AN, Song PH, McHugh M, Robbins J, Harrison MI. Highperformance work systems in health care management, part 2: qualitative evidence from five case studies. Health care management review. 2011;36(3):214-26.

15. Alavi SH, Marzban S, Gholami S, Najafi M, Rajaee R. How much is managers' awareness of evidence based decision making? Biomedical and Pharmacology Journal. 2015;8(2):1015-23.

16. Guo R, Berkshire SD, Fulton LV, Hermanson PM. Use of evidence-based management in healthcare administration decision-making. Leadership in Health Services. 2017;30(3):330-42.

17. Arndt M, Bigelow B, editors. Evidencebased management in health care organizations: A critique of its 
assumptions. Academy of Management. 2007;10(1):1-6

18. Champagne F, Lemieux-Charles L, Duranceau M-F, MacKean G, Reay $T$. Organizational impact of evidenceinformed decision making training initiatives: a case study comparison of two approaches. Implementation Science. 2014;9(1):53-59.

19. Alexander JA, Hearld LR, Jiang HJ, Fraser I. Increasing the relevance of research to health care managers: hospital CEO imperatives for improving quality and lowering costs. Health care management review. 2007;32(2):150-9.

20. Pfeffer J, Sutton RI. Profiting from evidence-based management. Strategy and Leadership. 2006;34(2):35-42.

21. Van der Heide I, van der Noordt M, Proper KI, Schoemaker C, van den Berg M, Hamberg-van Reenen HH. Implementation of a tool to enhance evidence-informed decision making in public health: identifying barriers and facilitating factors. Evidence \& Policy: A Journal of Research, Debate and Practice. 2016;12(2):183-97.

22. Oliver JD. Examining barriers of using evidence-based decision making by managers in practice: University of Prince Edward Island; 2013.

23. Axelsson R. Towards an evidence based health care management. The International journal of health planning and management. 1998;13(4):307-17.

24. Kovner AR. Evidence-based teaching: a course in health services management. The Journal of health administration education. 2003;20(4):235-42.

25. Pfeffer J, Sutton RI. Management halftruths and nonsense: How to practice evidence-based management. California Management Review. 2006;48(3):77$100+4$.
26. Rousseau DM. 2005 Presidential Address: Is There Such A Thing A "Evidence-Based Management"? Academy of Management The Academy of Management Review. 2006;31(2):256-69.

27. Briner RB, Denyer D, Rousseau DM. Evidence-based management: Concept cleanup time? Academy of Management Perspectives. 2009;23(4):19-32.

28. Barends E, Have ST, Huisman F. Learning from Other Evidence-Based Practices: The Case of Medicine. The Oxford Handbook of Evidence-Based Management. 2012; 2(1):25-42.

29. Wright AL, Zammuto RF, Liesch PW, Middleton S, Hibbert P, Burke J, et al. Evidence-based Management in Practice: Opening up the Decision Process, Decision-maker and Context. British Journal of Management. 2016;27(1):16178.

30. Moher D, Liberati A, Tetzlaff J, Altman DG. Preferred reporting items for systematic reviews and meta-analyses: the PRISMA statement. Annals of internal medicine. 2009;151(4):264-9.

31. O'Brien BC, Harris IB, Beckman TJ, Reed DA, Cook DA. Standards for reporting qualitative research: a synthesis of recommendations. Academic Medicine. 2014;89(9):1245-51.

32. Pace R, Pluye P, Bartlett G, Macaulay AC, Salsberg J, Jagosh J, et al. Testing the reliability and efficiency of the pilot Mixed Methods Appraisal Tool (MMAT) for systematic mixed studies review. International journal of nursing studies. 2012;49(1):47-53.

33. Malpass A, Shaw A, Sharp D, Walter F, Feder G, Ridd M, et al. "Medication career" or "moral career"? The two sides of managing antidepressants: a metaethnography of patients' experience of 
antidepressants. Social Science \& Medicine. 2009;68(1):154-68.

34. Dixon-Woods M, Booth A, Sutton AJ. Synthesizing qualitative research: a review of published reports. Qualitative Research. 2007;7(3):375-422.

35. Pope C, Ziebland S, Mays N. Analysing qualitative data. BMJ (Clinical research ed). 2000;320(7227):114-6.

36. Barends E, Villanueva J, Rousseau DM, Briner RB, Jepsen DM, Houghton E, Ten Have S. Managerial attitudes and perceived barriers regarding evidence-based practice: An international survey. PloS one. 2017;12(10):184-94.

37. Brownson RC, Reis RS, Allen P, Duggan $\mathrm{K}$, Fields $\mathrm{R}$, Stamatakis $\mathrm{KA}$, et al. Understanding administrative evidencebased practices: Findings from a survey of local health department leaders. American Journal of Preventive Medicine. 2014;46(1):49-57.

38. Charlier SD, Brown KG, Rynes SL. Teaching evidence-based management in MBA programs: What evidence is there? Academy of Management Learning and Education. 2011;10(2):222-36.

39. Guo R. Prediction of intention to use evidence-based management among healthcare administrators in the United States [D.H.A.]. Ann Arbor: Central Michigan University; 2015.

40. Guo R, Farnsworth TJ, Hermanson PM. Information Resources for Hospital Administrator Healthcare Management Decision-Making. Journal of hospital librarianship. 2015;15(3):274-83.

41. HakemZadeh F. Evidence-Based PolicyMaking in Canada. Canadian Journal of Administrative Sciences-Revue Canadienne Des Sciences De L Administration. 2014;31(3):214-5.

42. Jacob RR, Baker EA, Allen P, Dodson EA, Duggan K, Fields R, et al. Training needs and supports for evidence-based decision making among the public health workforce in the United States. BMC Health Services Research. 2014;14.

43. Niedźwiedzka BM. Barriers to evidencebased decision making among Polish healthcare managers. Health Services Management Research. 2003;16(2):106-15.

44. Workentine S. Understanding The Spread of Evidence-Informed Decision Making in a Government Health Department in Canada 2014.

45. Yousefi-Nooraie R, Dobbins M, Marin A. Social and organizational factors affecting implementation of evidence-informed practice in a public health department in Ontario: a network modelling approach. Implementation science : IS. 2014;9:29.

46. Amodeo M, Lundgren L, Beltrame CF, Chassler D, Cohen A, D'Ippolito M. Facilitating Factors in Implementing Four Evidence-Based Practices: Reports from Addiction Treatment Staff. Substance Use \& Misuse. 2013;48(8):600.

47. Bullock A, Morris ZS, Atwell C. Collaboration between health services managers and researchers: making a difference? Journal of health services research \& policy. 2012;17 Suppl 2:2-10.

48. Ellen ME, Leon G, Bouchard G, Lavis JN, Ouimet M, Grimshaw JM. What supports do health system organizations have in place to facilitate evidence-informed decision-making? A qualitative study. Implementation science : IS. 2013;8:84.

49. Ellen ME, Léon G, Bouchard G, Ouimet M, Grimshaw JM, Lavis JN. Barriers, facilitators and views about next steps to implementing supports for evidenceinformed decision-making in health systems: a qualitative study. Implementation Science. 2014;9.

50. Ferlie E, Sue Dopson, Louise Fitzgerald, Michael D Fischer, Jean Ledger, McGivern 
G. Getting Beyond The Tower Of Babel?: Disparate Evidence Based Management(s) in Health Care. Academy of Management Annual Meeting. 2012.

51. Francis-Smythe J, Robinson L, Ross C. The role of evidence in general managers' decision-making. Journal of General Management. 2013;38(4):3-21.

52. Jack SM, Dobbins M, Sword W, Novotna G, Brooks S, Lipman EL, et al. Evidenceinformed decision-making by professionals working in addiction agencies serving women: a descriptive qualitative study. Substance abuse treatment, prevention, and policy. 2011;6(1):1.

53. Kohn MK. Evidence Based Strategic Decision Making in Ontario Public Hospitals: University of Toronto; 2013.

54. Majdzadeh R, Yazdizadeh B, Nedjat S, Gholami J, Ahghari S. Strengthening evidence-based decision-making: is it possible without improving health system stewardship? Health Policy and Planning. 2012;27(6):499.

55. McBride LB. The role of evidence-based decision making in organizations: A phenomenological study [Ph.D.]. Ann Arbor: Capella University; 2015.

56. Peirson L, Ciliska D, Dobbins M, Mowat D. Building capacity for evidence informed decision making in public health: a case study of organizational change. $B M C$ Public Health. 2012;12:137.

57. Plath D. Organizational Processes Supporting Evidence-Based Practice. Administration in Social Work. 2013;37(2):171.

58. Plath D. Implementing Evidence-Based Practice: An Organisational Perspective.
British Journal of Social Work. 2014;44(4):905.

59. Richer MC, Dawes M, Marchionni C. Bringing knowledge to action in the context of a major organizational transition. The health care manager. 2013;32(1):4-12.

60. Schuller KA, Kash BA, Gamm LD. Studer Group ${ }^{\circledR}$ 's evidence-based leadership initiatives: Comparing success and sustainability in two health systems. Journal of Health Organization and Management. 2015;29(6):684-700.

61. Sosnowy CD, Weiss LJ, Maylahn CM, Pirani SJ, Katagiri NJ. Factors affecting evidence-based decision making in local health departments. American journal of preventive medicine. 2013;45(6):763-8.

62. Spiri WC, MacPhee M. The meaning of evidence-based management to Brazilian senior nurse leaders. Journal of nursing scholarship. 2013;45(3):265-72.

63. Armstrong R, Waters E, Moore L, Dobbins M, Pettman T, Burns C, et al. Understanding evidence: a statewide survey to explore evidence-informed public health decision-making in a local government setting. Implementation Science. 2014;9.

64. Martelli PF. An Argument for Knowledge Variety in Evidence-Based Management [Ph.D.]. Ann Arbor: University of California, Berkeley; 2012.

65. Yost J, Ciliska D, Dobbins M. Evaluating the impact of an intensive education workshop on evidence-informed decision making knowledge, skills, and behaviours: a mixed methods study. BMC Medical Education. 2014;14(1):13-22. 